\title{
A randomized controlled trial evaluating the effects of amlodipine on myocardial iron deposition in pediatric patients with thalassemia major
}

This article was published in the following Dove Press journal:

Drug Design, Development and Therapy

\author{
Arwa Khaled' \\ Hoda A Salem ${ }^{2}$ \\ Dina A Ezzat ${ }^{3}$ \\ Hadeel M Seif ${ }^{4}$ \\ Hoda Rabee ${ }^{5}$ \\ 'Department of Clinical Pharmacy, Beni- \\ Suef University Hospital, Beni-Suef \\ University, Beni Suef, 625I5, Egypt; \\ ${ }^{2}$ Department of Clinical Pharmacy, \\ Faculty of Pharmacy, Al-azhar University, \\ Girl Branch, Cairo, 12655, Egypt; \\ ${ }^{3}$ Department of Pediatrics and Pediatric \\ Hematology, Faculty of Medicine, Beni- \\ Suef University, Beni Suef, 12568, Egypt; \\ ${ }^{4}$ Department of Radiology, Faculty of \\ Medicine, Cairo University Cairo, Cairo \\ 8582, Egypt; ${ }^{5}$ Department of Clinical \\ Pharmacy, Faculty of Pharmacy, Beni-Suef \\ University, Beni-Suef, 625I4, Egypt
}

Background: Mortality rates increase due to iron deposition in the cardiac muscles of thalassemia major (TM) patients. Iron overload cardiomyopathy could be treated with a combination therapy of an iron chelator and an L-type calcium channel blocker. We designed a randomized controlled study to assess the potential of amlodipine, alongside chelation, in reducing myocardial iron concentration in TM patients compared with a placebo.

Objectives: This study aims to estimate the change in myocardial iron concentration (MIC) determined by magnetic resonance imaging after 6 months of treatment with amlodipine, as well as measuring the changes in the secondary outcomes (liver iron concentration (LIC), serum ferritin level (SF), and left ventricle ejection fraction (LVEF)) of study participants.

Methods: A single, randomized, placebo-controlled trial was performed in $40 \beta$ Thalassemia major patients aged between 6 and 20 years old, who received either oral amlodipine $2.5-5 \mathrm{mg}$ /day or a placebo, in addition to a Deferasirox chelation regimen in a $1: 1$ allocation ratio.

Results: After 6 months, a significant reduction was noted in the MIC of patients receiving amlodipine $(n=20)$, compared with the patients receiving the placebo $(n=20)$. At baseline, the mean was $0.76 \pm 0.11 \mathrm{mg} / \mathrm{g}$ dry weight, while at 6 months, the mean was $0.51 \pm 0.07 \mathrm{mg} / \mathrm{g}$ dry weight $(p<0.001)$. Also, there was a significant change in the myocardial T2* after 6 months; the amlodipine increased the myocardial $\mathrm{T} 2 *$ from $40.63 \pm 5.45 \mathrm{~ms}$ at baseline to 43.25 $\pm 5.35 \mathrm{~ms}(p<0.001)$. However, amlodipine did not significantly affect the secondary outcomes by the end of the study.

Conclusion: The addition of amlodipine to the standard chelation therapy in transfusiondependent thalassemia major patients improves myocardial iron overload without increasing the adverse effects.

Keywords: thalassemia major, amlodipine, magnetic resonance imaging, myocardial iron concentration

\section{Introduction}

Thalassemia syndromes are a heterogeneous group of hemoglobin disorders which exhibit decreased or absent production of normal globin chains. ${ }^{1}$

Chronic hemolytic anemia results from a defect in hemoglobin synthesis, which leads to the reduced synthesis of $\beta$-globin chains. Hemolytic anemia is characterized by its severity, its development during the first year of life and its requirement of life-long transfusion therapy. ${ }^{2}$ This hemoglobin disorder leads to $\beta$-Thalassemia, one of the most common inherited blood disorders of thalassemia births. ${ }^{1}$ Two clinical forms of $\beta$ thalassemia have been distinguished, depending on clinical severity: thalassemia major
Correspondence: Arwa Khaled

Clinical Pharmacist, Beni-Suef University

Hospital, I8-el Fondi st Mokbal, Beni Suef 62515, Egypt

Tel +20 10I 1332315

Email dr_arwakhaled@yahoo.com 
and thalassemia intermedia. $\beta$-Thalassemia major is the typical phenotype, arising either from homozygous or compound heterozygous defects. ${ }^{3,4}$ Transfusion-dependent Thalassemia patients receive more iron than is normal. This iron accumulation causes damage to various body organs, mainly the heart, which is very sensitive to iron toxicity, ${ }^{5}$ but also the liver and endocrine organs ${ }^{6}$ Heart failure due to myocardial siderosis is a result of the heart tissues' slow removal of excess iron. ${ }^{7-10}$ this is the major cause of death in transfusion-dependent thalassemia major patients. ${ }^{7}$

Therefore, myocardial iron measurement is an important step in determining the risk of cardiac complications. ${ }^{11}$ and in tailoring the appropriate iron-chelation treatment for transfused thalassemia major patients. ${ }^{4,12}$ Cardiac T2* magnetic resonance imaging (MRI), using an intense magnetic field, can successfully assess iron deposition in cardiac muscles. The values derived from "T2*MRI" are inversely proportional to tissue iron levels. Most recorded cases of heart failure in thalassemia patients to date have occurred in patients with very low T2* values. ${ }^{11,13,14}$ In particular, myocardial T2* values less than 20 milliseconds $(\mathrm{ms})$ indicate cardiac iron overload, while in severe cardiac iron overload, the T2* value is less than $10 \mathrm{~ms}^{4,15}$ Subsequently, the accuracy and reproducibility of T2*MRI measurements are important for the management of patients with iron overload cardiomyopathy.

Despite the availability of iron chelation therapy, ironmediated cardiac toxicity remains the leading cause of death in thalassemia major patients ${ }^{16}$ Although intense chelation can help many patients, depletion of the cardiac iron burden often takes years and mortality is high with incomplete compliance. ${ }^{17}$

Myocardial iron overload occurs when transferrin becomes saturated and iron is free rather than being regulated by transferrin-mediated uptake mechanisms under normal iron homeostasis. ${ }^{18}$ Previous studies in mice demonstrated that calcium channel blockers could be a means to remove iron from cardiac muscles. ${ }^{19-21}$

Amlodipine (AML) is a dihydropyridine calcium channel blocker that competitively inhibits the calcium channel to prevent calcium influx into the cell ${ }^{22}$ Its antioxidant property has been proven in various studies. ${ }^{23,24}$ fortunately, it is available at an affordable price that allows maximum compliance, which makes the drug highly suitable for our study. It is an orally administrated drug with a known safety profile in both children and adults.

Amlodipine's pharmacokinetic properties differ from the other nondihydropyridine CCBs, since amlodipine has a long half-life (35-50 hr.) compared with verapamil (3-7 hrs) or nitrendipine (2-5 hrs). The long half-life of Amlodipine decreases the time between dosage intervals and minimizes the large differences in peak to trough plasma concentration for a longer time. ${ }^{25}$

A limited number of human studies have shown a significant reduction in MIC after using amlodipine. ${ }^{26-28}$ Hence, we conducted a randomized, controlled-placebo study to demonstrate the efficacy of amlodipine, in addition to chelation therapy, in reducing myocardial iron concentration.

\section{Methods}

The study was designed as a single center, prospective randomized, placebo-controlled trial with allocation of a 1:1 ratio. The study was carried out in Beni-Suef University Hospital, Egypt. Thalassemia is prevalent in this city, as it serves neighboring villages where the disease is also common. Ethical committee approval (Ethical committee code: FWA00015574) and written informed consent from parents or caregivers were obtained before the start of the study. The clinical trial registry number is PACTR201902478249291. The trial was conducted in accordance with the Declaration of Helsinki.

Patients eligible for enrollment at baseline were male or female aged between 6 and 20 years old with $\beta$ Thalassemia major. Potential participants had been diagnosed with thalassemia major (TM), due to the presence of microcytic hypochromic anemia and hemoglobin electrophoresis. Additionally, patients who had been receiving regular blood transfusions during the past two years, with a serum ferritin (SF) level more than $1000 \mathrm{ng} / \mathrm{ml}$, were also considered. Participants were excluded if they were more than 20 years old, their SF was less than $1000 \mathrm{ng} / \mathrm{ml}$, they experienced heart failure (ejection fraction (EF) less than $30 \%$ ), they were contraindicated to undergo the MRI scan, or they were expected to change their chelation therapy regimen during the next 6 months.

Patients were invited to participate during a visit to the outpatient hematology clinic at Beni-Seuf University Hospital (BUH). Once they met the inclusion criteria and signed the consent form, blood samples were collected for hematological and chemical analysis, and MRI scans were performed.

After doing the MRI scans and other laboratory tests, patients were allocated into either the iron chelator (Deferasirox) plus amlodipine group (Norvasc; Pfizer $2.5 \mathrm{mg} /$ day for patients weighing less than $30 \mathrm{~kg}$ and $5 \mathrm{mg} /$ day for patients weighing more than $30 \mathrm{~kg})^{25}$ or the iron 
chelator Deferasirox placebo group (Exjade; Novartis 20 $40 \mathrm{mg} / \mathrm{kg} /$ day) for 6 months. ${ }^{29}$ The clinical pharmacist generated a computer list to randomly allocate the patients to either the drug or placebo group. The study medications were dispensed at each monthly visit and the patients were informed as to the group to which they were assigned.

\section{Magnetic resonance imaging (MRI)}

While all 40 TM patients were admitted to Beni-Suef University Hospital, the MRI scan were conducted at Al Kasr El-Aini Hospital, Egypt, where a four-element cardiac phased-array coil was used. Scans were synchronized to the cardiac cycle using standard ECG gating, according to a specific protocol for the measurement of heart $\mathrm{T} 2 *$, liver $\mathrm{T}^{*}$, MIC, and LIC. ${ }^{15}$

Participants were asked to take a single breath and hold it while a single $10 \mathrm{~mm}$ mid-ventricular slice was placed precisely mid-point between the base of the short axis and the head of the left ventricle (TE=2.6-18.8 ms, with $2.02 \mathrm{~ms}$ accretion).

For T2* and MIC analysis, a homogeneous full-thickness region of interest (ROI) was chosen in the septum ${ }^{30}$ The signal intensity of this region was measured for each of the images and the data were plotted against the TE to form an exponential decay curve. Cut-off points in this MRI instrument are as follows: Cardiac: normal $>20 \mathrm{~ms}$, mild: $14-20 \mathrm{~ms}$, moderate: 10-14 ms, severe $<10 \mathrm{~ms}$; Liver: normal $>6.3 \mathrm{~ms}$, mild: 2.8 $6.3 \mathrm{~ms}$, moderate: $1.4-2.7 \mathrm{~ms}$, severe $<1.4 \mathrm{~ms}^{12}$

The analysis was carried out on a PC using Thalassemia-Tools software. The measurements of the heart $\mathrm{T}^{*}$ and MIC were done according to the protocol developed by Carpenter et $\mathrm{al}^{6}{ }^{6}$ while the measurements of the liver T2* and LIC followed that of MW Garbowski. ${ }^{31}$

\section{Serum ferritin}

Serum was separated, labeled, and stored frozen at $-20^{\circ} \mathrm{C}$ and was measured by micro-particle enzyme immunoassay (Abbott AXSYM System).

\section{Efficacy assessment}

The primary trial endpoint was the change in the MIC and cardiac $\mathrm{T} 2 *$ after 6 months in either the placebo or treatment group. Efficacy data were analyzed as changes from core baseline to month 6 .

However, we considered the drug effective if there was a change in the MIC, rather than the $\mathrm{T} 2 *$, after the publication of a study done by Carpenter et al which showed a nonlinear correlation between $\mathrm{T}^{*}$ and $\mathrm{MIC}^{6}$ The use of both MIC and LIC allow for a precise measurement of the iron concentration in the tissues. ${ }^{29,32}$

The secondary trial endpoint noted potential changes in the LIC, liver T2*, SF, and LVEF after 6 months of treatment in either group.

\section{Safety assessment}

Participants and their parents were informed of the expected side effects of amlodipine, such as edema, dizziness and swollen ankles. Participants in both groups were monitored for adverse effects by a complete blood count (CBC) and physical examination during their routine visits to the hematology clinic. A minor change in the chelation therapy regimen was allowed during the study, particularly for participants with excess iron concentration. Participants were also educated about the importance of compliance with the medication.

\section{Statistical analysis}

Using G*Power software version 3.1.9.2 (Post hoc detection of power), we conducted a one-sided two sample Wilcoxon-Mann-Whitney $t$-test using the major variables. We calculated a sample size of 20 in each group (Amlodipine and placebo) achieving nearly 100\%, depending on effect size, with a significance level $(\alpha)$ of 0.05 , normality of data and 2-tailed analysis.

All the data were expressed as mean $\pm \mathrm{SD}$, with differences among the groups from baseline to 6 months compared for all continuous parameters. Independent t-tests were performed for the parametric variables and a Mann Whitney test for the non-parametric variables (particularly MIC, serum ferritin, LIC, liver T2*, myocardial T2*). The respective changes in the $\mathrm{MIC}$, LIC and serum ferritin within each group were not normally distributed, so we compared them using the Wilcoxon rank test and paired sample $t$-test for the normally distributed parameters. Statistical analysis was performed using the Statistical Package for Social Sciences (SPSS) computer software (version 22), IBM software, USA. Differences were considered statistically significant at $p<0.05$.

\section{Results}

\section{The baseline demographic and clinical characteristics}

The baseline demographic characteristics showed no significant differences between the amlodipine and the placebo groups, with the exception of the age at onset, liver 
$\mathrm{T} 2 *$ and liver iron concentration (LIC), with more iron deposition found in the livers of the placebo group patients (Table 1). Cardiac iron overload defined by $\mathrm{T} 2 * \leq 35 \mathrm{~ms}$ $(\mathrm{MIC} \leq 0.59 \mathrm{mg} / \mathrm{g})^{33,34}$ was observed in $50 \%$ of both the amlodipine and placebo group members at baseline. Patients flow-through is shown in Figure 1.

The initial MIC showed no statistically significant differences in the patients allocated to placebo or amlodipine treatment, with a mean \pm SD of $0.74 \pm 0.11 \mathrm{mg} / \mathrm{g}$ vs 0.76 $\pm 0.11 \mathrm{mg} / \mathrm{g}$, respectively $(P=0.87)$. The clinical characteristics of the study patients are shown in Table 1.

\section{Myocardial iron concentration}

The addition of amlodipine to the standard chelator therapy showed a significant reduction in the MIC, from 0.76 $\pm 0.11 \mathrm{mg} / \mathrm{g}$ at baseline to $0.51 \pm 0.07 \mathrm{mg} / \mathrm{g}$ after 6 months $(p<0.001)$ Figure 2. Also, a significant change in the myocardial T2* was noted after 6 months; the addition of amlodipine increased the myocardial T2* from $40.63 \pm 5.45 \mathrm{~ms}$ at baseline to $43.25 \pm 5.35 \mathrm{~ms}(p<0.001)$ (Table 2$)$.

The MIC significantly increased in patients receiving the placebo after 6 months of the trial, from $0.74 \pm 0.11 \mathrm{mg} / \mathrm{g}$ to $0.8 \pm 0.11 \mathrm{mg} / \mathrm{g}(p<0.001)$. Additionally, the myocardial T2* significantly decreased, from $53.23 \pm 6.61 \mathrm{~ms}$ at baseline to $52.99 \pm 6.6 \mathrm{~ms}$ after 6 months $(p=0.009)$ (Figure 3). The differences between the groups were significantly in favor of the amlodipine treatment group (Table 3).

\section{Serum ferritin}

The serum ferritin level did not change significantly in either of the two groups by the end of the study, measuring $1929 \pm 421.06 \mathrm{ng} / \mathrm{ml}$ for the amlodipine group and 2759 $\pm 340.73 \mathrm{ng} / \mathrm{ml}$ for the placebo group ( $p=0.925$ ).

\section{Liver iron concentration}

The absolute changes in LIC were not significant in either the placebo or the amlodipine groups. However, a significant difference was found in the relative liver $\mathrm{T} 2 *$ after 6 months, as the liver T2* decreased in the placebo group from a mean of $20.19 \pm 2.21 \mathrm{~ms}$ to $20.00 \pm 2.23 \mathrm{~ms}$ $(p=0.004)$ (Table 4), while no significant change in the relative LIC was noted after 6 months.

\section{Left ventricular ejection fraction}

The mean \pm SD left ventricular ejection fraction remained stable and within the normal range after 6 months of treatment for both groups. The amlodipine group baseline measure was $62.28 \pm 0.79 \%$, while at month 6 , it was 62.40 $\pm 0.76 \%$, an absolute change of $-0.125 \pm 0.64 \quad(p=0.398)$. For the placebo group, the baseline was $63.40 \pm 0.55 \%$, while at month 6 , it was $63.10 \pm 0.57 \%$, with an absolute change of $0.3 \pm 1.12(p=0.249)$.

\section{Adverse effects}

A significant difference was noted between the amlodipine and placebo groups regarding side effects. More patients in the placebo group experienced gastro-intestinal (G.I) upset- a total of 14 patients $(70 \%)$ compared to six patients $(30 \%)$ in the amlodipine group. Four $(20 \%)$ of the amlodipine participants complained of dizziness and 3 (15\%) experienced swollen ankles. No cases of palpitation or hypotension were reported in the treatment group (Figure 4).

Minor changes in chelation therapy were allowed during the study, particularly for patients with severe iron overload or those who experienced side effects from the treatment. This consisted of minor adjustments in the Deferasirox dose. No patients switched to other chelators during the 6 months of the study.

\section{Discussion}

Cardiomyopathy resulting from iron overload is still a common cause of morbidity and mortality in transfusiondependent TM patients. ${ }^{2,35}$ Despite treatment with the most commonly used iron chelators and regular blood transfusions, a significant proportion of $\beta$-TM patients have myocardial iron loading. Moreover, chronic iron overload can lead to several diseases, including cirrhosis, diabetes, neurodegenerative disease, and endocrine disorders ${ }^{36}$ Therefore, a logical step toward reducing cardiac mortality is optimizing the treatment of myocardial siderosis.

In this study, we found that the addition of oral Amlodipine to the standard chelation therapy reduced the myocardial iron concentration in transfusion-dependent thalassemia major patients. This combination is more effective than iron chelators alone. After 6 months of treatment, the myocardial iron concentration decreased significantly ( $p$-value <0.001). This is explained by the mechanism of iron uptake in the heart tissues, which is mediated by the L-type calcium channels. The relative improvement of MIC in the amlodipine patients was consistent with a previous study by Fernandes et al (2016), also indicating a significant reduction in the MIC and improvement in the heart T2* after treatment with amlodipine in 57 patients older than 6 years of age. ${ }^{26,37}$ 
Table I Baseline demographic and clinical characteristics

\begin{tabular}{|c|c|c|c|c|c|}
\hline \multirow{2}{*}{\multicolumn{2}{|c|}{ Variable }} & \multirow[t]{2}{*}{ Placebo } & \multirow[t]{2}{*}{ Amlodipine } & \multicolumn{2}{|c|}{ Base line characteristic } \\
\hline & & & & t. or $z$ or $X^{2}$ values & $P$-value \\
\hline \multicolumn{2}{|l|}{ Age (years) } & $|3.40 \pm 1.0|$ & $12.50 \pm 1.02$ & 0.627 & 0.535 \\
\hline \multicolumn{2}{|l|}{ Weight/kg } & $38.08 \pm 2.53$ & $36.23 \pm 2.80$ & $\underline{0.490}$ & 0.627 \\
\hline \multicolumn{2}{|l|}{ Height $/ \mathrm{m}$} & $1.39 \pm 0.03$ & $1.35 \pm 0.04$ & $\underline{0.888}$ & 0.380 \\
\hline \multicolumn{2}{|c|}{ Pretransfusional.Hg (g/dl) } & $7.47 \pm 0.37$ & $7.24 \pm 0.27$ & $\underline{0.510}$ & 0.613 \\
\hline \multicolumn{2}{|c|}{ No. of blood transfusion/life } & $159.85 \pm 15.16$ & $181.90 \pm 17.46$ & 0.953 & 0.346 \\
\hline \multicolumn{2}{|c|}{ Deferasirox dose $\mathrm{mg} / \mathrm{kg} /$ day } & $1151.25 \pm 111.72$ & $|200.00 \pm||5.2|$ & $\underline{0.304}$ & 0.763 \\
\hline \multicolumn{2}{|l|}{ BMI } & $19.17 \pm 0.61$ & $19.48 \pm 0.72$ & 0.027 & 0.978 \\
\hline \multicolumn{2}{|c|}{ Age at onset (months) } & $12.85 \pm 2.37$ & $8.25 \pm 1.4 \mid$ & 2.032 & 0.042 \\
\hline \multicolumn{2}{|c|}{ Duration of disease (years) } & $12.39 \pm 1.03$ & $11.91 \pm 1.02$ & 0.352 & 0.725 \\
\hline Gender & $\begin{array}{l}\text { Male } \\
\text { Female }\end{array}$ & $\begin{array}{l}9(45 \%) \\
\text { II (55\%) }\end{array}$ & $\begin{array}{l}8(40 \%) \\
12(60 \%)\end{array}$ & 0.102 & 0.749 \\
\hline Splenectomy & $\begin{array}{l}\text { No } \\
\text { Yes }\end{array}$ & $\begin{array}{l}18(90 \%) \\
2(10 \%)\end{array}$ & $\begin{array}{l}17(85 \%) \\
3(15 \%)\end{array}$ & 0.230 & 0.632 \\
\hline
\end{tabular}

Note: The Italic numbers are $X^{2}$. The underlined values refer to the $t$-test. The other numbers are $z$ values.

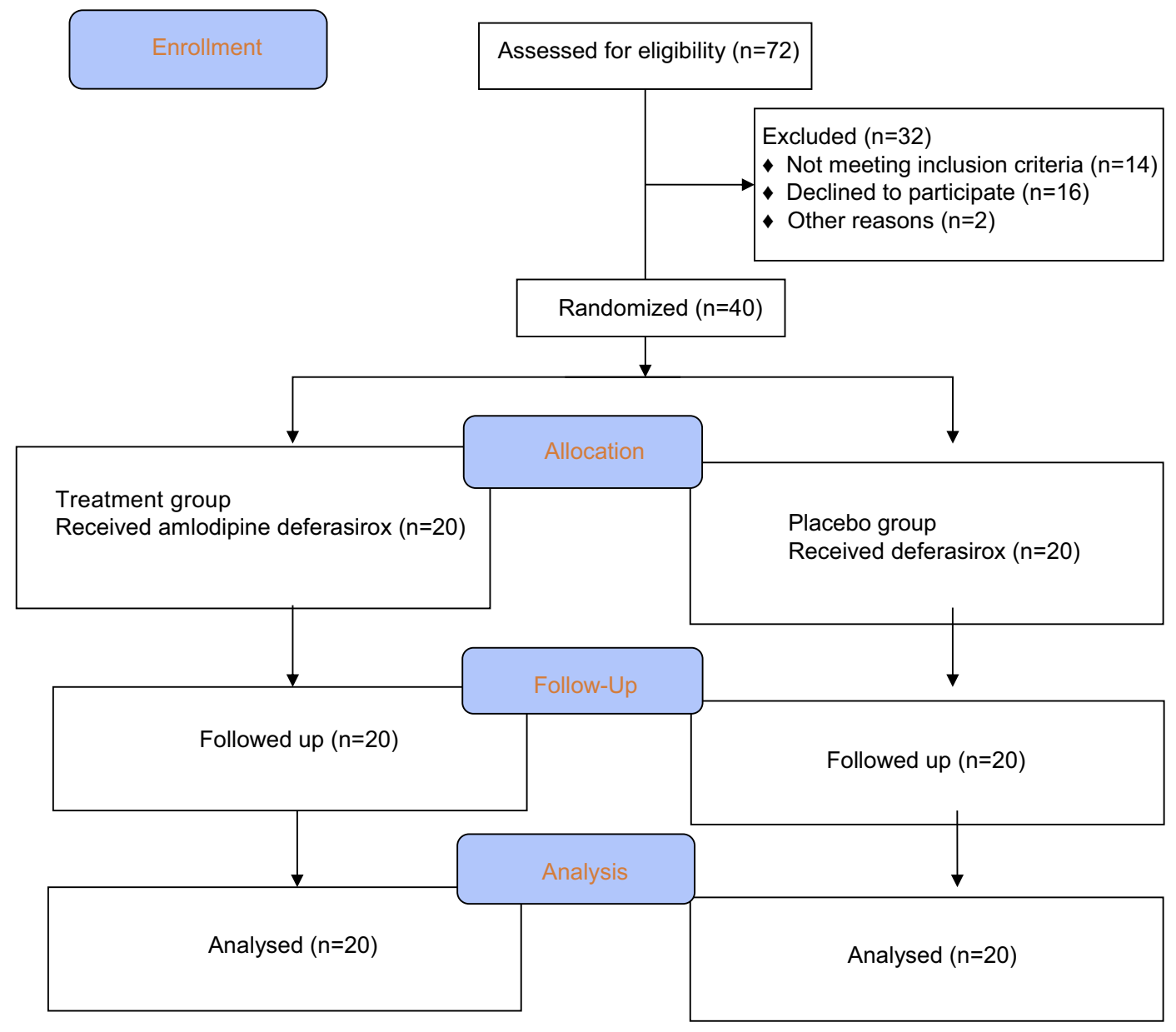

Figure I Consort diagram detailing the study subjects. 


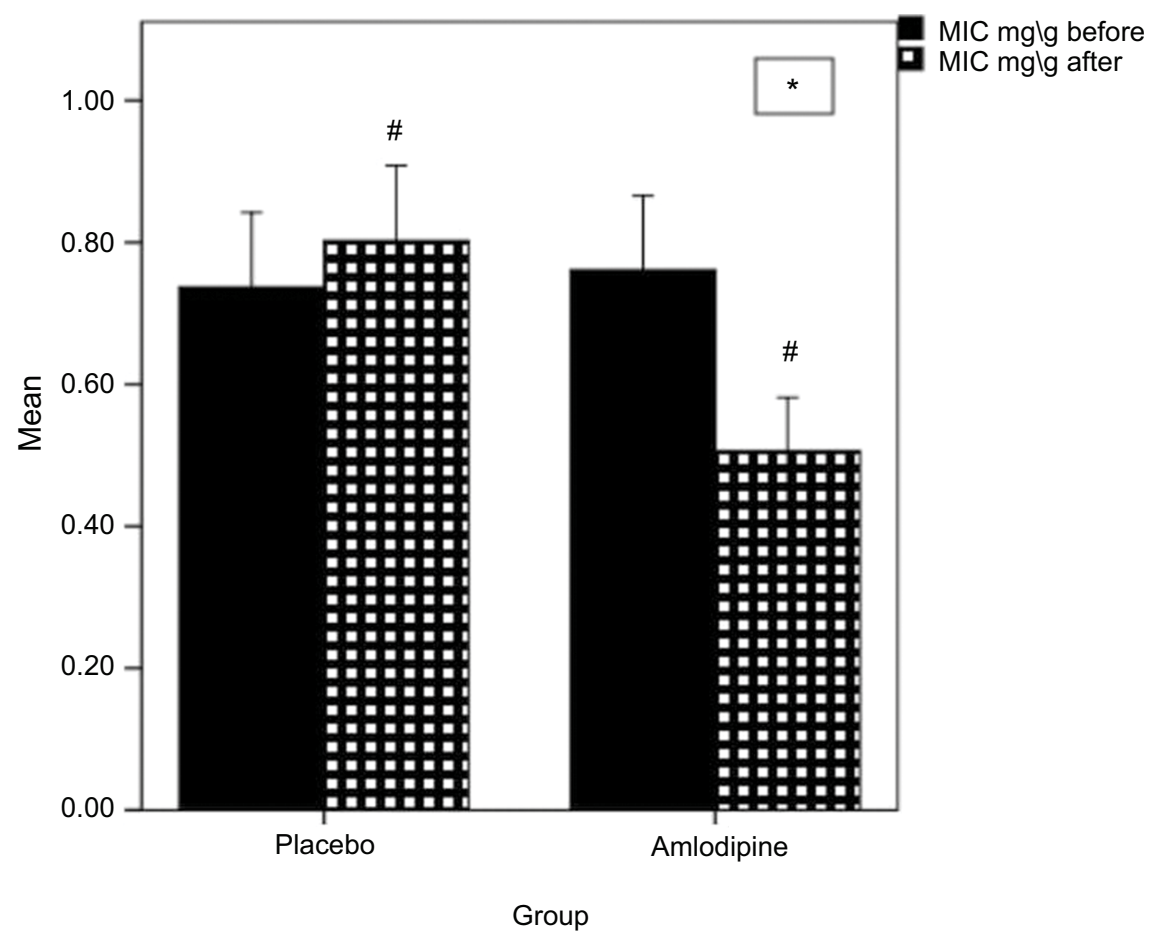

Figure 2 Mean change in MIC.

Note: *Significantly different from placebo (significant difference between Amlodipine and placebo). " Significantly different from before or baseline (significant difference between before and after).

Table 2 Comparison between placebo and amlodipine in before (Base line characteristics) and after treatments

\begin{tabular}{|l|l|l|l|l|l|}
\hline Variable & Placebo & Amlodipine & t. or z values & P-value & $\begin{array}{l}\text { Amlodipine + or - by \% regarding } \\
\text { Placebo }\end{array}$ \\
\hline S.F before, $\mathrm{ng} / \mathrm{ml}$ & $2752.20 \pm 342.47$ & $2949.85 \pm 420.90$ & 0.027 & 0.978 & 7.18 \\
S.F after, ng/ml & $2759.00 \pm 340.73$ & $1929.00 \pm 421.06$ & 0.095 & 0.925 & -30.08 \\
Liver T2* before, ms & $20.19 \pm 2.21$ & $35.25 \pm 5.57$ & 2.340 & 0.019 & 74.59 \\
Liver T2* after, ms & $20.00 \pm 2.23$ & $35.38 \pm 5.59$ & 2.381 & 0.017 & 76.90 \\
MIC before, mg/g & $0.74 \pm 0.1 \mathrm{I}$ & $0.76 \pm 0.1 \mathrm{I}$ & $\underline{0.162}$ & 0.872 & 2.70 \\
MIC after, mg/g & $0.80 \pm 0.1 \mathrm{I}$ & $0.51 \pm 0.07$ & $\underline{2.282}$ & 0.028 & -36.25 \\
LIC before, mg/g & $2.74 \pm 0.34$ & $1.64 \pm 0.55$ & 3.395 & 0.001 & -40.15 \\
LIC after, mg/g & $2.77 \pm 0.34$ & $1.63 \pm 0.55$ & 3.423 & 0.001 & -41.16 \\
Myocardial T2* before, ms & $53.23 \pm 6.61$ & $40.63 \pm 5.45$ & 1.894 & 0.058 & -23.67 \\
Myocardial T2* after, ms & $52.99 \pm 6.60$ & $43.26 \pm 5.35$ & 1.447 & 0.148 & -18.36 \\
LVEF before,\% & $63.40 \pm 0.55$ & $62.28 \pm 0.79$ & $\underline{1.171}$ & 0.249 & -1.70 \\
LVEF after, \% & $63.10 \pm 0.57$ & $62.40 \pm 0.76$ & $\underline{0.737}$ & 0.466 & $-1.1 \mathrm{I}$ \\
\hline
\end{tabular}

Note: The underlined values refer to the $t$-test. The other numbers are $z$ values.

Abbreviations: MIC, myocardial iron concentration; LIC, liver iron concentration; MRI, magnetic resonance imaging; ms, milliseconds; SF, serum ferritin; LVEF, left ventricle ejection fraction.

In addition, our results revealed a significant improvement in myocardial $\mathrm{T} 2 *$ after treatment with amlodipine, from $40.63 \pm 5.45 \mathrm{~ms}$ at baseline to $43.25 \pm 5.35 \mathrm{~ms}$. This finding is in accordance with the recent work by Eghbali et al, which indicated that myocardial $\mathrm{T}^{*}$ significantly changed after 1 year of treatment, from $21.9 \mathrm{~ms}$ to $24.5 \mathrm{~ms} .{ }^{28}$ Another study conducted by Fernandes et al (2014) found an increase of $30 \%$ in the myocardial T2* after 1 year of treatment with amlodipine. ${ }^{27,37}$

However, no change was noted in the LIC and hepatic T2* after 6 months of treatment with amlodipine. We suggest this is due to the fact that iron deposition in the liver tissues does not depend on active uptake by voltage-gated calcium channels; therefore, blocking or opening the calcium channels would not 


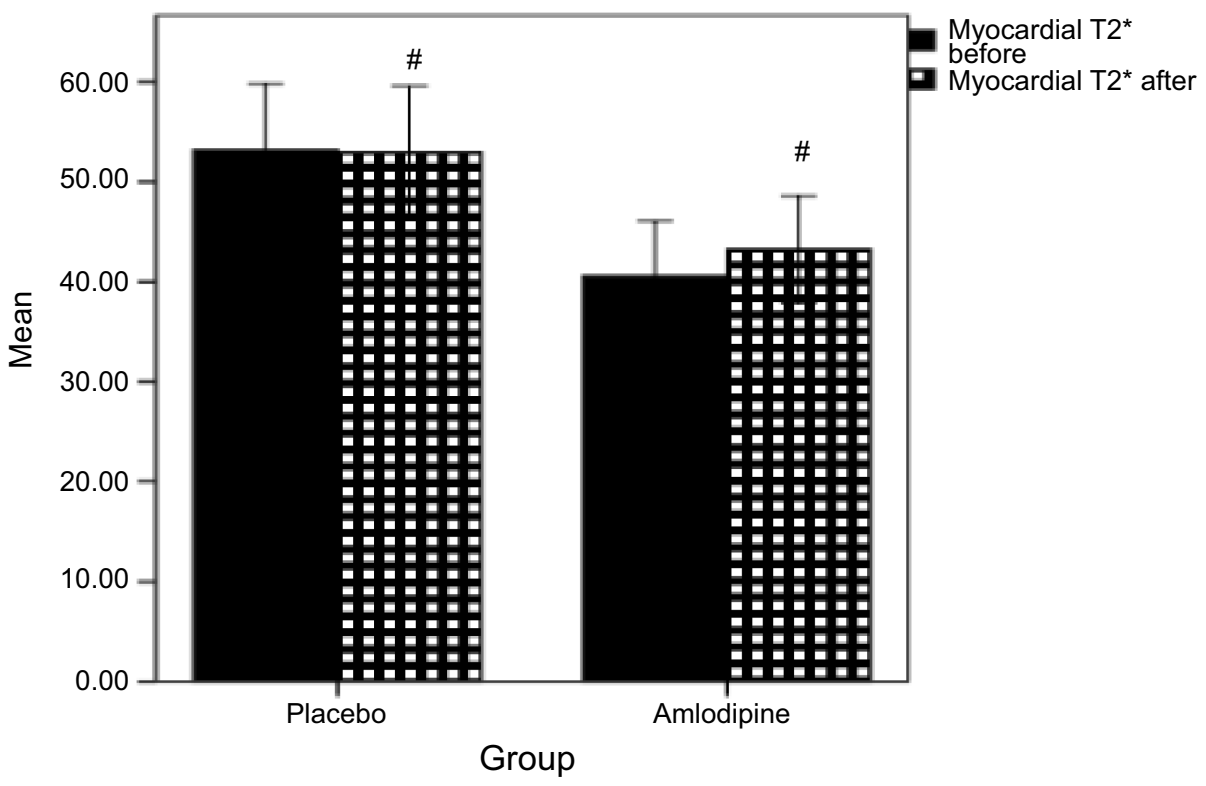

Figure 3 Mean change in Myocardial T2*.

Note: *Significantly different from placebo (significant difference between Amlodipine and placebo). ${ }^{\#}$ Significantly different from before or baseline (significant difference between before and after).

Table 3 Outcomes at 6 months (Amlodipine group)

\begin{tabular}{|l|l|l|l|l|l|}
\hline Variable & Before & After & t. or z values & $\boldsymbol{p}$-value & After + or - by \% regarding base line \\
\hline MIC mg/g & $0.76 \pm 0.11$ & $0.51 \pm 0.07$ & 7.532 & $<0.001$ & -32.8947 \\
Myocardial T2* MRI, ms & $40.63 \pm 5.45$ & $43.26 \pm 5.35$ & 3.811 & $<0.001$ & $6.473,049$ \\
LIC, mg/g & $1.64 \pm 0.55$ & $1.63 \pm 0.55$ & 1.123 & 0.261 & -0.61 \\
Hepatic T2* MRI, ms & $35.25 \pm 5.57$ & $35.38 \pm 5.59$ & 1.427 & 0.154 & 0.37 \\
SF, ng/ml & $2949.85 \pm 420.90$ & $1929.00 \pm 421.06$ & 1.364 & 0.172 & -34.6068 \\
LVEF,\% & $62.28 \pm 0.79$ & $62.40 \pm 0.76$ & 0.865 & 0.398 & 0.19 \\
\hline
\end{tabular}

Note: The underlined values refer to the $t$-test. The other numbers are $z$ values.

Table 4 Outcomes at 6 months (placebo group)

\begin{tabular}{|l|l|l|l|l|l|}
\hline Variable & Before & After & t. or z values & p-value & After + or - by \% regarding base line \\
\hline MIC, mg/g & $0.74 \pm 0.1 \mathrm{I}$ & $0.80 \pm 0.1 \mathrm{I}$ & 4.429 & $<0.00 \mathrm{I}$ & $8.108,108$ \\
Myocardial T2* MRI, ms & $53.23 \pm 6.6 \mathrm{I}$ & $52.99 \pm 6.60$ & 2.599 & 0.009 & $-0.45,087$ \\
LIC, mg/g & $2.74 \pm 0.34$ & $2.77 \pm 0.34$ & 1.1312 & 0.190 & $1.09489 \mathrm{I}$ \\
Hepatic T2* MRI, ms & $20.19 \pm 2.21$ & $20.00 \pm 2.23$ & 2.917 & 0.004 & $-0.94,106$ \\
SF, ng/ml & $2752.20 \pm 342.47$ & $2759.00 \pm 340.73$ & 0.946 & 0.344 & $0.247,075$ \\
LVEF, \% & $63.40 \pm 0.55$ & $63.10 \pm 0.57$ & 1.189 & 0.249 & $-0.47,319$ \\
\hline
\end{tabular}

Note: The underlined values refer to the t-test. the other numbers are $\mathrm{z}$ values.

Abbreviations: MIC, myocardial iron concentration; LIC, liver iron concentration; MRI, magnetic resonance imaging; ms, milliseconds; SF, serum ferritin; LVEF, left ventricle ejection fraction.

affect the iron uptake into the liver tissue. This is in accordance with the studies done by Fernandes et al (2016) and Eghbali et al (2017). ${ }^{26,28}$

Although no significant change was found in the serum ferritin level by the end of the study, the median of SF was kept below $2500 \mathrm{ng} / \mathrm{ml}$, which is associated with increased morbidity and mortality. ${ }^{38,39}$ Thus, the serum ferritin level is not a good indicator of myocardial iron deposition. This is supported by many previous studies which have demonstrated a weak correlation between plasma ferritin levels and cardiac T2*MRI. ${ }^{12,40}$

Only $12.5 \%$ of our patients underwent a splenectomy. This low rate may be due to the early diagnosis of the disease and the availability of blood products in Egypt. 


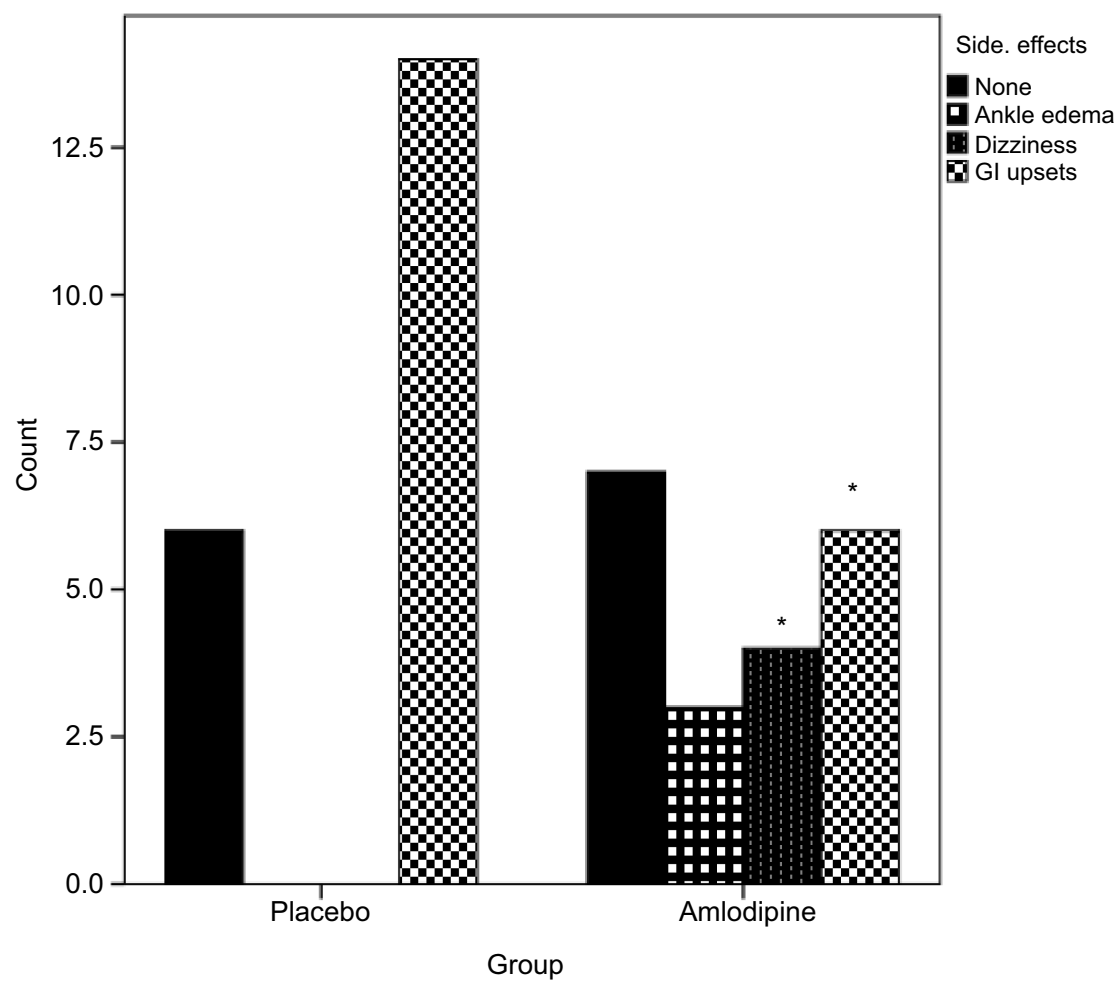

Figure 4 Untoward effects of treatments; gastrointestinal upset was the most common adverse effect in both groups*. Note: *Significantly different from placebo (significant difference between Amlodipine and placebo).

In this study, the amlodipine had no serious adverse effects (Figure 4), which is consistent with the known safety profile of amlodipine in previous studies. ${ }^{41,42}$

A limited number of clinical trials in our country have demonstrated the efficacy of amlodipine in reducing myocardial iron concentration. Therefore, this study focused on the efficacy of amlodipine on myocardial iron deposition in pediatric patients with thalassemia major in Egypt.

\section{Study limitations}

Although our findings indicated that the addition of amlodipine to the standard chelator therapy results in a significant reduction in the myocardial iron concentration, our study included a short observation period and a small sample size. The effects of long-term treatment with amlodipine and the use of different iron chelators should be assessed by other studies with larger sample sizes. Since the removal of the accumulated iron from heart tissues is a slow process, we suggest long-term controlled studies be established to strengthen the evidence demonstrating the clinical benefits of amlodipine alongside an iron chelation regimen.

We did not find any significant clinical change in the LVEF after six months of treatment with amlodipine. This outcome may also require a longer-term study or the recruitment of patients with reduced ejection fraction from the start of the study.

\section{Conclusion}

In conclusion, it is clinically beneficial to add amlodipine to the standard chelation therapy in patients with transfusion-dependent thalassemia major, as the amlodipine results in a significant reduction in the myocardial iron concentration.

\section{Disclosure}

The authors report no conflicts of interest in this work.

\section{References}

1. Origa R. $\quad \beta$-Thalassemia. Genet Med. 2017;19(6):609-619. doi:10.1038/gim.2016.173

2. Kremastinos DT, Farmakis D, Aessopos A, et al. $\beta$-thalassemia cardiomyopathy: history, present considerations, and future perspectives. Circ Hear Fail. 2010;3(3):451-458. doi:10.1161/CIRCHEARTFAILURE. 109.913863

3. Hakeem GLA, Mousa SO, Moustafa AN, Mahgoob MH, Hassan EE. Health-related quality of life in pediatric and adolescent patients with transfusion-dependent $\beta$-thalassemia in upper Egypt (single center study). Health Qual Life Outcomes. 2018;16(1):59. doi:10.1186/ s12955-018-0893-z 
4. Tanner MA, Galanello R, Dessi C, et al. A randomized, placebo-controlled, double-blind trial of the effect of combined therapy with deferoxamine and deferiprone on myocardial iron in thalassemia major using cardiovascular magnetic resonance. Circulation. 2007;115(14):18761884. doi:10.1161/CIRCULATIONAHA.106.648790

5. Gujja P, Rosing DR, Tripodi DJ, Shizukuda Y. Iron overload cardiomyopathy: better understanding of an increasing disorder. $J$ Am Coll Cardiol. 2010;56(13):1001-1012. doi:10.1016/j.jacc.2010.03.083

6. Carpenter J, He T, Kirk P, et al. NIH public access. Circulation. 2012;123 (14):1519-1528. doi:10.1161/CIRCULATIONAHA.110.007641.On

7. Pennell DJ, Porter JB, Piga A, et al. Sustained improvements in myocardial $\mathrm{T} 2 *$ over 2 years in severely iron-overloaded patients with beta thalassemia major treated with deferasirox or deferoxamine. Am J Hematol. 2015;90(2):91-96. doi:10.1002/ajh.23876

8. Gillin JC, van Kammen DP, Graves J, Murphy D. Differential effects of D- and L-amphetamine on the sleep of depressed patients. Life Sci. 1975;17(8):1233-1240. doi:10.3324/haematol.2011.049957

9. Noetzli LJ, Carson SM, Nord AS, et al. Longitudinal analysis of heart and liver iron in thalassemia major Longitudinal analysis of heart and liver iron in thalassemia major. October. 2008;112(7):2973-2978. doi:10.1182/blood-2008-04-148767

10. Aydinok Y, Porter JB, Piga A, et al. Prevalence and distribution of iron overload in patients with transfusion-dependent anemias differs across geographic regions: results from the CORDELIA study. Eur $J$ Haematol. 2015;95(3):244-253. doi:10.1111/ejh.12487

11. Pennell DJ, Berdoukas V, Karagiorga M, et al. Randomized controlled trial of deferiprone or deferoxamine in beta-thalassemia major patients with asymptomatic myocardial siderosis. Blood. 2006;107(9):3738-3744. doi:10.1182/blood-2005-07-2948

12. Soltanpour MS, Davari K. The correlation of cardiac and hepatic hemosiderosis as measured by $\mathrm{T} 2 * \mathrm{MRI}$ technique with ferritin levels and hemochromatosis gene mutations in Iranian patients with beta thalassemia major. Oman Med J. 2018;33(1):48-54. doi:10.5001/omj.2018.09

13. Pepe A, Positano V, Santarelli MF, et al. Multislice multiecho T2* cardiovascular magnetic resonance for detection of the heterogeneous distribution of myocardial iron overload. J Magn Reson Imaging. 2006;23:662-668. doi:10.1002/jmri.20566

14. Habib G, Bucciarelli-Ducci C, Caforio ALP, et al. Multimodality imaging in restrictive cardiomyopathies: an EACVI expert consensus document In collaboration with the "Working Group on myocardial and pericardial diseases" of the European Society of Cardiology Endorsed by The Indian Academy of Echocardiography. Eur Heart J Cardiovasc Imaging. 2017;18(10):1090-1121. doi:10.1093/ehjci/jex034

15. He T, Gatehouse PD, Smith GC, Mohiaddin RH, Pennell DJ, Firmin DN. Myocardial T2* measurements in iron overloaded thalassemia. Magn Reson Med. 2008;60(5):1082-1089. doi:10.1002/mrm.21744.Myocardial

16. Borgna-Pignatti C, Rugolotto S, De Stefano P, et al. Survival and complications in patients with thalassemia major treated with transfusion and deferoxamine. Haematologica. 2004;89(10):1187-1193.

17. Davis BA, O'Sullivan C, Jarritt PH, Porter JB. Value of sequential monitoring of left ventricular ejection fraction in the management of thalassemia major. Blood. 2004;104(1):263-269. doi:10.1182/blood2003-08-2841

18. Wijarnpreecha K, Kumfu S, Chattipakorn SC, Chattipakorn N. Cardiomyopathy associated with iron overload: how does iron enter myocytes and what are the implications for pharmacological therapy? Hemoglobin. 2015;39(1):9-17. doi:10.3109/03630269.2014.987869

19. Kumfu S, Chattipakorn S, Chinda K, Fucharoen S, Chattipakorn N. T-type calcium channel blockade improves survival and cardiovascular function in thalassemic mice. Eur J Haematol. 2012;88(6):535548. doi:10.1111/j.1600-0609.2012.01779.x

20. Lopin KV, Gray IP, Obejero-Paz CA, Thevenod F, Jones SW. Fe2+ Block and Permeation of CaV3.1 (1G) T-Type Calcium Channels: candidate Mechanism for Non-Transferrin-Mediated Fe2+ Influx. Mol Pharmacol. 2012;82(6):1194-1204. doi:10.1124/mol.112.080184
21. Oudit GY, Trivieri MG, Khaper N, Liu PP, Backx PH. Role of L-type Ca2 + channels in iron transport and iron-overload cardiomyopathy. $J \mathrm{Mol}$ Med. 2006;84(5):349-364. doi:10.1007/s00109-005-0029-x

22. Lee YJ, Park HH, Koh SH, Choi NY, Lee KY. Amlodipine besylate and amlodipine camsylate prevent cortical neuronal cell death induced by oxidative stress. J Neurochem. 2011;119(6):1262-1270. doi:10.1111/ j.1471-4159.2011.07529.x

23. Rosenkranz AC, Lob H, Breitenbach T, Berkels R, Roesen R. Endothelial antioxidant actions of dihydropyridines and angiotensin converting enzyme inhibitors. Eur J Pharmacol. 2006;529(1-3):5562. doi:10.1016/j.ejphar.2005.10.046

24. Salehi I, Mohammadi M, Mirzaei F, Soufi FG. Amlodipine attenuates oxidative stress in the heart and blood of high-cholesterol diet rabbits. Cardiovasc J Afr. 2012;23(1):18-22. doi:10.5830/CVJA-2010-091

25. Buck ML, Pharm D. A Monthly Newsletter for Health Care Professionals from the. Schizophrenia. 2008;14(1):6-9. doi:10.13422/j. cnki.syfjx.2012.02.067

26. Fernandes JL, Loggetto SR, Ver MPA, et al. A randomized trial of amlodipine in addition to standard chelation therapy in patients with thalassemia major. Blood. 2016;128(12):1555-1562. doi:10.1182/ blood-2016-06-721183. The

27. Shakoor A, Zahoor M, Sadaf A, et al. Effect of 1-type calcium channel blocker (amlodipine) on myocardial iron deposition in patients with thalassaemia with moderate-to-severe myocardial iron deposition: protocol for a randomised, controlled trial. BMJ Open. 2014;4(12). doi:10.1136/bmjopen-2014-005360

28. Eghbali A, Kazemi H, Taherahmadi H, Ghandi Y, Rafiei M, Bagheri B. A randomized, controlled study evaluating effects of amlodipine addition to chelators to reduce iron loading in patients with thalassemia major. Eur J Haematol. 2017;577-581. doi:10.1111/ejh. 12977

29. Pennell DJ, Porter JB, Cappellini MD, et al. Continued improvement in myocardial $\mathrm{T} 2 *$ over two years of deferasirox therapy in $\beta$-thalassemia major patients with cardiac iron overload. Haematologica. 2011;96(1):48-54. doi:10.3324/haematol.2010.031468

30. Ghugre NR, Enriquez CM, Coates TD, Nelson MD Jr, Jr N, Wood JC. Improved R2* measurements in myocardial iron overload. $J$ Magn Reson Imaging. 2006;23(1):9-16. doi:10.1002/jmri.20467. Improved

31. Garbowski MW, Carpenter JP, Smith G, et al. Biopsy-based calibration of $\mathrm{T} 2 *$ magnetic resonance for estimation of liver iron concentration and comparison with R2 Ferriscan. J Cardiovasc Magn Reson. 2014;16(1):1-11. doi:10.1186/1532-429X-16-40

32. Pepe A, Meloni A, Rossi G, et al. Cardiac and hepatic iron and ejection fraction in thalassemia major: multicentre prospective comparison of combined Deferiprone and Deferoxamine therapy against Deferiprone or Deferoxamine Monotherapy. J Cardiovasc Magn Reson. 2013;15(1):1-11. doi:10.1186/1532-429X-15-1

33. Positano V, Pepe A, Santarelli MF, et al. Standardized T2* map of normal human heart in vivo to correct T2* segmental artefacts. NMR Biomed. 2007;20:578-590. doi:10.1002/nbm.1121

34. Ramazzotti A, Pepe A, Positano V, et al. Standardized T2* map of a normal human heart to correct $\mathrm{T} 2 *$ segmental artefacts; myocardial iron overload and fibrosis in thalassemia intermedia versus thalassemia major patients and electrocardiogram changes in thalassemia major patients. Hemoglobin. 2008;32:97-107. doi:10.1080/036302 60701879514

35. Pennell DJ, Udelson JE, Arai AE, et al. Cardiovascular function and treatment in $\beta$-thalassemia major: a consensus statement from the american heart association. Circulation. 2013;128(3):281-308. doi:10.1161/ CIR.0b013e31829b2be6

36. Wongjaikam S, Kumfu S, Chattipakorn SC, Fucharoen S, Chattipakorn N. Current and future treatment strategies for iron overload cardiomyopathy. Eur J Pharmacol. 2015;765:86-93. doi:10.1016/j.ejphar.2015.08.017 
37. Sadaf A, Hasan B, Das JK, Colan S, Alvi N. Calcium channel blockers for preventing cardiomyopathy due to iron overload in people with transfusion-dependent beta thalassaemia. Cochrane Database Syst Rev. 2018;2018(7). doi:10.1002/14651858.CD011626.pub2

38. Opelz G, Wujciak T. The New England Journal of Medicine Downloaded from nejm.org at ALBERT EINSTEIN COLLEGE OF MEDICINE on November 22, 2011. For personal use only. No other uses without permission. Copyright (C) 1994 Massachusetts Medical Society. All rights reserved. Nejm. 2011;331(1):5-9. doi:10.1056/NEJM199409293311301

39. Guillaume T, Rubinstein DB, Sysman M. The journal of the American Society of Hematology. J Am Soc Hematol. 2016;92 (5):1471-1491. doi:10.1007/s00542-013-2002-x
40. Eghbali A, Taherahmadi H, Shahbazi M, Bagheri B, Ebrahimi L. Association between serum ferritin level, cardiac and hepatic T2-star $\{\mathrm{MRI}\}$ in patients with major $\beta$-thalassemia. Iran J Ped Hematol Oncol. 2014;4(1):17-21.

41. Triggle DJ. Calcium channel antagonists: clinical uses-Past, present and future. Biochem Pharmacol. 2007;74(1):1-9. doi:10.1016/j. bcp.2007.01.016

42. DeWitt CR, Waksman JC. Pharmacology, pathophysiology and management of calcium channel blocker and beta-blocker toxicity. Toxicol Rev. 2004;23(4):223-238.

\section{Publish your work in this journal}

Drug Design, Development and Therapy is an international, peerreviewed open-access journal that spans the spectrum of drug design and development through to clinical applications. Clinical outcomes, patient safety, and programs for the development and effective, safe, and sustained use of medicines are a feature of the journal, which has also been accepted for indexing on PubMed Central. The manuscript management system is completely online and includes a very quick and fair peer-review system, which is all easy to use. Visit http://www. dovepress.com/testimonials.php to read real quotes from published authors. 